\title{
THE USE OF A MICHELSON INTERFEROMETER ON A COARSELY STABILIZED SPACECRAFT TO OBTAIN HIGH RESOLUTION (0.1 ̊) OVER 1300-3300 $\AA$
}

\author{
D. D. CLARK \\ Space 1, Ministry of Technology, London, U.K.*
}

The spectral resolution of the prism or grating spectrograph depends on its angular dispersion which, in a space environment, implies that resolution also depends on the accuracy of pointing of the space platform.

The achievement of high pointing accuracy is not easy and requires a complex, and hence costly, attitude control and space reference system aboard the spacecraft. It would be preferable if discrimination were not based on angular dispersion since angle is the one parameter to which the spacecraft is most sensitive. By employing a Michelson interferometer the independent variable, by means of a Fourier transform, becomes one of linear displacement. The problem now is the accurate measurement of length, independent of angle, and the task of reconstructing the spectrum is done on the computers on the ground using the modern fast Fourier transform.

In this way the attainment of high resolving power is effectively decoupled from the pointing accuracy. Both the movement control and parallelism of the mirror in the interferometer are achieved by means of the interference fringes from a high coherence reference beam, using established methods.

The Michelson is prefered to the Fabry-Pérot because the tolerance for surface flatness of the operating surfaces is much less severe and it is easier to obtain a wide range. With a $\mathrm{LiF}$ or $\mathrm{MgF}_{2}$ dividing plate it is hoped to cover the range 1300 to $3300 \AA$.

There remain however the problems of acquisition and background. The interferometer operates with a wide field of view, e.g. a resolving power of 25000 can be achieved within a field one degree wide. To observe point sources in a field of this width would limit observable magnitudes to about $M_{v}=4$. However if stars are acquired in the wide field their location with reference to spacecraft axes could be determined by an image dissector tube operating off the zero order light. By using image dissector tubes also as detectors and subsequently reading off the signal only from the 'instantaneous' photo-cathode area, the excess background of the wide field is eliminated. In this way it should be possible to reach much fainter magnitudes.

Confirmation of stellar identity could be achieved by transmitting to the ground the 'spacecraft' co-ordinates of the three brightest stars in the field.

It is more practical, in addition to saving observing and computing time, to divide

* Now with Department of Trade and Industry, London. 
the spectral output into several bands of equal wave number which are observed separately yet simultaneously. The observing times are then comparable with a conventional spectrograph of the same aperture.

\section{DISCUSSION}

B. Bates: 1. What is the proposed method for the linear displacement of the movable mirror?

2. The Fabry-Pérot spectrometer would also only need a guidance of approx. 30 arc min for a spectral resolving power of 25000 .

D. D. Clark: This has not yet been decided but will probably be either by moving coil or linear motor. The problems of the Michelson interferometer are similar to those of the Fabry-Pérot, but the Fabry-Pérot is more sensitive to angle and the conditions for flatness of the surfaces are more severe than for the Michelson. The Michelson can also operate over a wide spectral range which the Fabry-Pérot cannot.

G. Courtès: The merit of this experiment is certainly that it is the only spectrometer giving a resolution of $0.1 \AA$ for a pointing accuracy of the order of $30^{\prime}$.

D. D. Clark: This is quite correct. The feature of the Michelson is that it undertakes the task of achieving high resolution and allows the recording to be undertaken by a low resolution system and makes small demands on the spacecraft stabilisation and pointing subsystems.

P. Stettler: Do you scan step by step or continuously?

D. D. Clark: Continuously with good constancy of speed. 